\title{
Laser Ablation Molecular Isotopic Spectrometry of carbon isotopes
}

\author{
Alexander A. Bol'shakov ${ }^{1}$, Xianglei Mao ${ }^{2}$, Jinesh Jain $^{3}$, Dustin L. McIntyre ${ }^{3}$, Richard E. Russo ${ }^{1,2}$ \\ ${ }^{1}$ Applied Spectra, Inc., Fremont, CA 94538, USA \\ ${ }^{2}$ Lawrence Berkeley National Laboratory, Berkeley, CA 94720, USA \\ ${ }^{3}$ National Energy Technology Laboratory, Pittsburgh, PA 15236, USA
}

\section{ABSTRACT}

Quantitative determination of carbon isotopes using Laser Ablation Molecular Isotopic Spectrometry (LAMIS) is described. Optical emission of diatomic molecules $\mathrm{CN}$ and $\mathrm{C}_{2}$ is used in these measurements. Two quantification approaches are presented: empirical calibration of spectra using a set of reference standards and numerical fitting of a simulated spectrum to the experimental one. Formation mechanisms of $\mathrm{C}_{2}$ and $\mathrm{CN}$ in laser ablation plasma are briefly reviewed to provide insights for implementation of LAMIS measurements. A simulated spectrum of the ${ }^{12} \mathrm{C}_{2}$ Swan system was synthesized using four constituents within $473.5-476.5 \mathrm{~nm}$. Simulation included three branches of ${ }^{12} \mathrm{C}_{2}(1-0)$, branches $\mathrm{R}(0-0)$ and $\mathrm{R}(1-1)$, and branch $\mathrm{P}(9-8)$ of ${ }^{12} \mathrm{C}_{2}$. Spectral positions of the tail lines in $\mathrm{R}(0-0)$ and $\mathrm{R}(1-1)$ were experimentally measured, since they were not accurately known before. The Swan band (1-0) of the isotopologue ${ }^{13} \mathrm{C}^{12} \mathrm{C}$ was also simulated. Fitting to the experimental spectrum yielded the ratio ${ }^{13} \mathrm{C} /{ }^{12} \mathrm{C}=1.08 \%$ in a 
good agreement with measurements by isotope ratio mass spectrometry. LAMIS promises to be useful in coal, oil and shale exploration, carbon sequestration monitoring, and agronomy studies.

\section{INTRODUCTION}

Since the beginning of the industrial revolution the carbon dioxide $\left(\mathrm{CO}_{2}\right)$ levels in the atmosphere have been on the rise and causing noticeable climate change. This increase is mostly due to burning of fossil fuels (i.e., coal, oil, and natural gas), which emit $\mathrm{CO}_{2}$ into the air. High concentrations of $\mathrm{CO}_{2}$ can pose an environmental hazard and can be dangerous to human health. In addition, an introduction of massive amounts of $\mathrm{CO}_{2}$ into the seas can cause acidification, alter water chemistry, and affect the life cycles of many marine organisms, particularly those at the lower end of the food chain [1].

Geologic sequestration of $\mathrm{CO}_{2}$ has the potential to achieve a significant reduction in the anthropogenic $\mathrm{CO}_{2}$ emissions. The process relies on capturing the $\mathrm{CO}_{2}$ from industrial/electrical power generation gas streams, compressing it, and pumping it into storage formations as a supercritical fluid for permanent storage [2,3]. If a leakage pathway develops the $\mathrm{CO}_{2}$ will potentially escape the containment formation causing elevated levels of the gas in atmosphere. To ascertain if the increased levels of $\mathrm{CO}_{2}$ are due to leakage from the storage sites the measurement of $\mathrm{CO}_{2}$ concentration alone may not be sufficient [4].

Carbon isotope ratios allow an investigator to potentially determine the origin of $\mathrm{CO}_{2}$ and can distinguish between different sources of feedstock for power plants [5,6]. The $\mathrm{CO}_{2}$ captured from 
power plants and injected underground has unique isotope ratios as compared to other sources and could be identical to the coal used in a particular power plant. Since coal obtained from different sources is likely to have different carbon isotope ratios, by knowing the isotopic fingerprints of each coal reservoir scientists can determine if the increased $\mathrm{CO}_{2}$ levels in the atmosphere are from the carbon storage sites. Establishing the isotope ratios for the coal used in power plants would allow for the leakage to be easily identified. Therefore, a need arises to quickly and accurately measure the carbon isotope ratios for coal reservoirs, which will not only aid in identifying the leakage source but will also be likely of interest to sequestration site operators and regulators.

Laser Ablation Molecular Isotopic Spectrometry (LAMIS) is a new optical technique for direct isotopic analysis of solid samples in ambient air [7,8]. LAMIS utilizes spectra of diatomic molecules formed in laser ablation plumes in air. Carbon isotope detection in LAMIS has been achieved using $\mathrm{C}_{2}$ and $\mathrm{CN}$ emission [7-10]. This technique is similar to Laser Induced Breakdown Spectroscopy (LIBS), although LAMIS measures molecular spectra appearing usually at longer delays after an ablation pulse than what is used in LIBS analysis. Molecular emissions yield isotopic information that is relatively easy to acquire. A spectrometer with modest spectral resolution can be suitable for measuring molecular isotopic spectra. Hence, LIBS and LAMIS techniques can be accomplished on the same instrument. Elemental and isotopic measurements can be performed with minimal sample preparation and benefits of rapid chemical mapping and depth profiling at high spatial definition, in real time at atmospheric pressure. Industrial, laboratory, and field operations are possible, potentially at a standoff distance to the sample [11]. 
The ability of combined LIBS and LAMIS for simultaneous elemental and isotopic analysis makes them a useful tool for carbon sequestration monitoring. Real-time monitoring of carbon isotope ratios can lead to a more thorough study and effective way to monitor success of carbon sequestration. LAMIS measurement avoids use of expensive mass spectrometers that require deep vacuum and time consuming sample preparation. In the past, optical emission analyses using the spectra of isotopologue molecules $\mathrm{C}_{2}, \mathrm{CN}, \mathrm{CO}$ and $\mathrm{N}_{2}$ were performed in biochemical and agrochemical studies with stable isotope labels [12,13]. Recently, spectra of $C_{2}$ were used to infer rotational temperature as a correction factor for improvement of LIBS precision in coal analysis [14]. Emission intensities of $\mathrm{CN}$ and $\mathrm{C}_{2}$ were also used to correct for matrix effects in LIBS analysis of coal [15].

This paper describes a LAMIS method for determination of carbon isotopes in coal and graphite samples using $\mathrm{CN}$ and $\mathrm{C}_{2}$ spectra. LAMIS will be expedient in investigating whether the increased levels of atmospheric $\mathrm{CO}_{2}$ originated from the coal mines or coal combustion plants. Establishing the carbon isotope ratios of parent coal mines could develop an isotope library for different coal reservoirs and will aid in identifying the potential environmental problems associated with the subsurface carbon sequestration. Variation of the natural isotopic ratio ${ }^{13} \mathrm{C} /{ }^{12} \mathrm{C}$ in different materials ranges from $0.96 \%$ to $1.15 \%$. These variations can be measured using LAMIS. In general, the paper is aimed at practical applications and analysis of geological samples, while significant part of this work is devoted to accurate characterization of the background in ${ }^{12} \mathrm{C}_{2}$ molecular spectrum, which is critical for measurements of the minor isotope ${ }^{13} \mathrm{C}$. 
Formation of $\mathrm{C}_{2}$ and $\mathrm{CN}$ species in laser ablation plasma

Different kinetic behavior was observed in laser ablation spectra of native molecular fragments vaporized from organic samples and diatomic molecules formed by association of atomized matter with constituents of ambient air [16,17]. Multiple plasma chemical reactions occurring in ablation plumes with different rates often result in non-stoichiometric distribution of species in laser ablation plasma. Hence, understanding of molecular formation mechanisms and proper selection of experimental conditions are very important for analytical implementation of LAMIS.

In a previous work [9], we concluded that atomization in a plasma plume from solid benzoic acid samples was only partial, revealing preferential ruptures of specific carbon bonds even at relatively high laser irradiance of about $5 \cdot 10^{10} \mathrm{~W} / \mathrm{cm}^{2}$ at $1064 \mathrm{~nm}$. Fragmentation of benzene rings into ${ }^{12} \mathrm{C}_{2}$ dimers observed at delays $1-8 \mu$ s after laser pulse was more effective than formation of molecules ${ }^{13} \mathrm{C}^{12} \mathrm{C}$ involving ${ }^{13} \mathrm{C}$ atom from a carboxylic-acid group ${ }^{13} \mathrm{COOH}$. As a consequence, determined isotopic ratios ${ }^{13} \mathrm{C} /{ }^{12} \mathrm{C}$ deviated significantly from the stoichiometric ratio in the sample. The latter effect was observed relatively close to the sample surface $(<2 \mathrm{~mm})$ and therefore, these results can be attributed to preferential evaporation of the native ${ }^{12} \mathrm{C}_{2}$ molecular fragments from a molten spot formed on the surface after laser ablation pulse. At longer distances from the sample $(2-5 \mathrm{~mm})$ and delays greater than $4 \mu \mathrm{s}$, the observed isotopic ratio ${ }^{13} \mathrm{C} /{ }^{12} \mathrm{C}$ was close to the real ratio in the sample [10]. 
Serrano et al. [18] systematically studied an influence of the original molecular structure on the optical emission in laser ablation plasmas of organic solids. They found that electronic distribution within the original organic molecule in the sample has a strong impact on the degree of atomization versus fragmentation during laser ablation. Strengths of various carbon bonds, aromatic structures, functional groups and isomers in the original sample have distinct effects on formation of plasma-phase species. Particularly, this complex interplay of processes influences the resulting optical emission from $\mathrm{C}_{2}$ and $\mathrm{CN}$ radicals in ablation plumes.

Laser pulse duration, irradiance and wavelength also have apparent effects on plume dynamics. Longer laser wavelength (e.g., $1064 \mathrm{~nm}$ ) facilitates ejection of polyatomic clusters in a photothermal regime of ablation, while use of laser wavelength at $266 \mathrm{~nm}$ or less can result in direct photochemical breaking of bonds [19], particularly because photon at $266 \mathrm{~nm}$ has energy $4.66 \mathrm{eV}$ that is larger than $\mathrm{C}-\mathrm{C}$ bond of $3.59 \mathrm{eV}$. Therefore, in photothermal ablation the gasphase $\mathrm{C}_{2}$ dimers evaporated as native fragments can dominate over those formed by molecular association of atomized carbon. Contrary, a photochemical regime of ablation can result in the opposite relationship with a portion of $\mathrm{C}_{2}$ formed by association of atomized carbon being larger than that of the vaporized native $\mathrm{C}_{2}$ fragments.

At near-threshold ablation (laser irradiance $10^{8}-10^{9} \mathrm{~W} / \mathrm{cm}^{2}$ ) of graphite in vacuum, the vapor in the plume dominated by $\mathrm{C}_{2}$ and $\mathrm{C}_{3}$ fragments which were directly ejected from ablated graphite as the intact native molecules [20]. Once released from melted graphite surface into the gas phase, they could dissociate only through collisions occurring infrequently in vacuum. At laser irradiance $4 \cdot 10^{8} \mathrm{~W} / \mathrm{cm}^{2}$ in helium $(1.3 \mathrm{kPa})$, no atomic carbon emission from graphite 
ablation was detected but $\mathrm{C}_{2}$ emission was clearly observed [21]. This is because evaporation of carbon in the molecular versus atomic form is thermodynamically favored. If helium pressure increased up to $80 \mathrm{kPa}$, collisions in the plume cause sublimation of ejected soot particles that becomes another channel for the formation of $\mathrm{C}_{2}$ and $\mathrm{C}_{3}$ [22].

With the increasing ablation laser irradiance $\left(>7 \cdot 10^{9} \mathrm{~W} / \mathrm{cm}^{2}\right)$, molecular emission usually does not considerably change while atomic carbon emission increases dramatically indicating that the prevailing fraction of the plume becomes atomized into free carbon atoms and atomic ions [16]. During ablation in ambient atmospheric air, carbon atomization is also aided by bouncing supersonic shock waves produced after a laser pulse [23]. The laser ablation plume expansion causes a snowplough effect that compresses and moves the background gas in front of the plume, if pressure of the ambient gas is above $1 \mathrm{kPa}$ [24]. In the latter case, most part of the plume is filled with ablated matter from the sample, but ambient gas can anyway entrain through vortexes near the sample surface [25].

Spectra of $\mathrm{CN}$ are readily observed during laser ablation of carbon-based samples in air, even if no nitrogen is present in the sample. Multiple reactions of ablated carbon with atmospheric nitrogen occur in plasma plumes. Several competing pathways of $\mathrm{CN}$ formation exist including ionic, atomic and molecular reactions. When a snowplough effect dominates, $\mathrm{CN}$ can only form at the front of the propagating shockwave [24]. Reactions $\mathrm{C}+\mathrm{N}_{2} \rightarrow \mathrm{CN}+\mathrm{N}$ and $\mathrm{C}_{2}+\mathrm{N}_{2} \rightarrow$ $2 \mathrm{CN}$ are both endothermic with activation energies of $1.9 \mathrm{eV}$ and $1.8 \mathrm{eV}$, respectively [26]. Required initial heating can be obtained from either a forceful shockwave or gas-phase absorption of the ablating laser beam energy. Revealing the latter effect, the direction of $\mathrm{CN}$ 
formation can be inclined toward the incident laser beam, while $\mathrm{C}_{2}$ radicals propagate either uniformly in a hemi-sphere or preferentially perpendicular to the sample surface regardless of the angle of the incident laser beam [27]. Density of ejected carbon decreases in time and so does $\mathrm{CN}$, usually with some delay after $\mathrm{C}_{2}$. The higher binding energy of $\mathrm{CN}$ molecule $(7.8 \mathrm{eV})$ relative to that of $\mathrm{C}_{2}(6.2 \mathrm{eV})$ increases the chances of $\mathrm{CN}$ survival in the plasma plume at longer delays and distances.

Using optical time-of-flight spectroscopy, Al-Shboul et al. observed fast and slow components of both $\mathrm{C}_{2}$ and $\mathrm{CN}$ emission in a plume expanding from graphite into ambient nitrogen at $0.13 \mathrm{kPa}$ [28]. The experiments attested that a fast component was due to initially accelerated $\mathrm{C}^{+}$ions, which underwent recombination with electrons and association into molecules forming "clouds" of excited $\mathrm{C}_{2}$ and $\mathrm{CN}$ at distances about $6 \mathrm{~mm}$ from the sample surface via multi-body reactions at a plume front: $2 \mathrm{C}^{+}+2 \mathrm{e}^{-} \rightarrow \mathrm{C}_{2}$ and $2 \mathrm{C}^{+}+2 \mathrm{e}^{-}+\mathrm{N}_{2} \rightarrow 2 \mathrm{CN}$. The slower component in $\mathrm{C}_{2}$ emission $(1-10 \mu \mathrm{s}$ ) was closer to the surface (within $\sim 5 \mathrm{~mm}$ ) and was attributed to association of relatively slow neutral carbon atoms accompanied by a longer tail resulting from collisional dissociation of polyatomic carbon clusters.

Plasma chemistry in the gas phase of an ablation plume is obviously complicated (125 species undergoing 1509 processes were included in a model for ablation of organic samples [29]). There are many participating mechanisms that can exchange roles at different times and local parts of the plume. The presented here brief review of molecular formation mechanisms facilitates basic understanding and thus, enables informed realization of accurate LAMIS measurements of carbon isotopic ratios using $\mathrm{C}_{2}$ and $\mathrm{CN}$ spectra. 


\section{EXPERIMENTAL DETAILS}

A lamp pumped, Q-switched Nd:YAG laser (Quantel USA Inc.) operating at $1064 \mathrm{~nm}$ with a pulse energy $\sim 150 \mathrm{~mJ}$ and a pulse duration of approximately 4 ns was used to ablate the samples. The laser beam of $6 \mathrm{~mm}$ in diameter was focused onto the sample with a fused silica lens (focal distance $50 \mathrm{~mm}$ ) to a spot diameter of $\sim 250 \mu \mathrm{m}$, either in open air or in a cylindrical quartz chamber flushed with argon or helium. Laser pulse repetition frequency was $10 \mathrm{~Hz}$. A second lens (diameter $50 \mathrm{~mm}$ ) was used to collect the laser-induced plasma emission in the direction perpendicular to the ablating laser beam. All measurements were performed at atmospheric pressure.

Ablation plasma plumes were imaged 1:1 onto an entrance slit of one of the two spectrometers. One of them was the IsoPlane SCT-320 spectrograph with 320-mm focal length (Acton/Princeton Instruments) and interchangeable gratings of 3600 or $2400 \mathrm{gr} / \mathrm{mm}$. The other one was Horiba JY-1250M spectrograph with 1250-mm focal length and a grating of 3600 gr/mm, which was used for high resolution measurements. The spectral resolution was about $0.01 \mathrm{~nm}$ in the latter case. Both spectrographs were fitted with Intensified Charge-Coupled Devices (ICCD) as a detector. The model used was PI-MAX2 (Princeton Instruments) featuring an imaging array $1024 \times 1024$ of $13-\mu \mathrm{m}$ pixels with the Unigen intensifier. The spectrum acquisition gate widths and delays were optimized to measure molecular emission from $\mathrm{C}_{2}$ and $\mathrm{CN}$, while minimizing intensity of atomic and continuum radiation. The collected spectra were 
averaged over several hundreds of laser pulses. Pre-ablation by 10 pulses was applied at every new sampling location to remove possible surface contamination from the samples.

Natural and isotope-enriched powdered substances were obtained from commercial sources, then pressed by a 7-ton press into pellets with a diameter $10 \mathrm{~mm}$. Enriched in ${ }^{13} \mathrm{C}$ powders of amorphous carbon, decanoic acid and benzamide were used in this study. Natural and ${ }^{13} \mathrm{C}$ enriched powders of decanoic acid were mixed in different proportions to prepare a set of reference standards for isotopic calibration with ${ }^{13} \mathrm{C}$ atomic fraction: 1.2, 1.5, 2.0, 5.0 and $9.9 \%$. Enriched in ${ }^{13} \mathrm{C}$ amorphous carbon was mixed with natural graphite powder to prepare $5 \%{ }^{13} \mathrm{C}$ amorphous carbon pellet. Depleted in ${ }^{13} \mathrm{C}$ glucose $\left(99.9 \%{ }^{12} \mathrm{C}_{6} \mathrm{H}_{12} \mathrm{O}_{6}\right)$ was used to study the spectral background under molecular spectrum of ${ }^{13} \mathrm{C}^{12} \mathrm{C}$. Moreover, enriched in ${ }^{15} \mathrm{~N}$ powder of benzamide was used to illustrate a possibility of simultaneous measurements of ${ }^{13} \mathrm{C}$ and ${ }^{15} \mathrm{~N}$ using LAMIS. Several natural samples of coal, graphite and diamond were tested to demonstrate consistent molecular spectra obtained from different samples. Similarly, a pelletized reference material SARM-18 (MINTEK, South Africa) was also used for this purpose.

\section{RESULTS AND DISCUSSION}

Several organic and inorganic materials were ablated to measure emission of diatomic radicals at the conditions optimized for LAMIS. Our previous measurements demonstrated that abundances of individual isotopes in material composition can be measured by LAMIS at least down to $\sim 1 \%$, using quantitatively calibrated molecular emission. Rare carbon isotope ${ }^{13} \mathrm{C}$ can be detected in sub-natural concentrations [8]. Moreover, carbon and nitrogen isotopes can be 
determined simultaneously using spectra of $\mathrm{CN}$ isotopologues. This possibility was already presented in our earlier publications on LAMIS measurements $[7,8,30]$.

To demonstrate simultaneous isotopic measurements, we ablated natural graphite and two pellets of benzamide enriched in either ${ }^{13} \mathrm{C}$ or ${ }^{15} \mathrm{~N}$. Using a spectral interval of $411-422 \mathrm{~nm}$, we measured the vibrational band progression $\mathrm{B}^{2} \Sigma^{+} \rightarrow \mathrm{X}^{2} \Sigma^{+}$with changing vibrational quantum number $\Delta v=-1$ of ${ }^{12} \mathrm{C}^{14} \mathrm{~N},{ }^{13} \mathrm{C}^{14} \mathrm{~N}$, and ${ }^{12} \mathrm{C}^{15} \mathrm{~N}$ radicals. With the experimental resolution of $\sim 0.06 \mathrm{~nm}$, the rotational structure of these bands was only partially resolved in our measurements, but isotopic shifts between $\mathrm{CN}$ isotopologues were clearly observed [8]. Measuring ${ }^{15} \mathrm{~N}$ required a helium flow purge to preclude nitrogen entrainment from air. The spectra were acquired using a $10 \mu$ s time gate delayed after the laser pulse by $1.5 \mu \mathrm{s}$. The delay optimized for these measurements was the shortest among all other experiments reported here because $\mathrm{CN}$ radicals are in general promptly formed in laser ablation, and because plasma in helium has lower temperature and recombines faster than plasma ignited in air or argon.

Another band progression of $\mathrm{CN} \mathrm{B}^{2} \Sigma^{+} \rightarrow \mathrm{X}^{2} \Sigma^{+}$with the opposite change in vibrational quantum numbers $\Delta v=+1$ was measured within $354-362 \mathrm{~nm}$ (Fig. 1) using a $3600 \mathrm{gr} / \mathrm{mm}$ grating in IsoPlane-320 spectrograph at the resolution of $\sim 0.04 \mathrm{~nm}$. Isotopic differences in the band heads were well resolved but their rotational structure was not. An advantage of using these bands over those with $\Delta v=-1$ is that the spectral peaks with $\Delta v=+1$, attributed to the minor isotopes ${ }^{13} \mathrm{C}$ and ${ }^{15} \mathrm{~N}$ occur at low background, beyond the sharp edge of the ${ }^{12} \mathrm{C}^{14} \mathrm{~N}(1-0)$ band head at $359.04 \mathrm{~nm}$. Only a weak band $\mathrm{B} \rightarrow \mathrm{X}(9-8)$ of the major isotopologue ${ }^{12} \mathrm{C}^{14} \mathrm{~N}$ has its peak nearby at $360.30 \mathrm{~nm}$. The band heads of $\mathrm{B} \rightarrow \mathrm{X}(1-0)$ transitions of both isotopologues ${ }^{12} \mathrm{C}^{15} \mathrm{~N}$ and 
${ }^{13} \mathrm{C}^{14} \mathrm{~N}$ occur at 359.43 and $359.57 \mathrm{~nm}$, respectively [31]. As noted before, the progression $\Delta v=-$ 1 requires multivariate calibration, because the bands from minor isotopes can be hidden within unresolved rotational lines of the major ${ }^{12} \mathrm{C}^{14} \mathrm{~N}$ bands [8]. In contrast, spectral data of the progression $\Delta v=+1$ can be quantified using either univariate or multivariate calibration. Multivariate regression is still preferable because unresolved or partially-resolved data can be utilized in addition to the resolved features (as confirmed for boron isotopes [32]). Such supplemental information can be contained in unresolved and overlapping R-branches of the $\mathrm{CN}$ bands seen in Fig. 1 below $358 \mathrm{~nm}$.

The results of calibrating LAMIS spectra of $\mathrm{CN}$ molecule using a multivariate partial least squares regression (PLSR) procedure for quantitative determination of ${ }^{13} \mathrm{C}$ content in solid samples are presented in Fig. 2. Reference samples were prepared by blending powders of natural and ${ }^{13} \mathrm{C}$-enriched decanoic acid. A natural ${ }^{13} \mathrm{C}$ atomic fraction was assumed as $1.07 \%$, and additional five blended samples were made nominally at 1.2, 1.5, 2.0, 5.0 and $9.9 \%$. The $\mathrm{CN}$ $\mathrm{B} \rightarrow \mathrm{X}(\Delta v=+1)$ spectra were collected in the interval 354-362 nm, similar to those shown Fig. 1. The error bars in Fig. 2 correspond to standard deviation of a single measurement within 10 replicates, while each measurement was made by accumulating spectra from 100 laser pulses. Each replicate signal was measured at a new sampling location but accumulation was performed at the same ablation spot. The natural ${ }^{13} \mathrm{C}$ atomic fraction was recalculated from these measurements as $(1.09 \pm 0.14) \%$ corresponding to the relative standard deviation of $12.8 \%$. Averaging over 10 measurements (each one made of 100-pulse accumulations) reduces an estimated random error of the mean ${ }^{13} \mathrm{C}$ fraction down to $\pm 0.044 \%$. 
Quantification of data plotted in Fig. 2 was performed by measuring spectra of known reference samples and applying PLSR to relate them to the nominal abundances of isotopes in the sample. This is an empirical method that alleviates errors attributed to possible isotope fractionation in the ablation plumes. Moreover, $\mathrm{CN}$ spectra in these experiments were less prone to isotope fractionation than those of $\mathrm{C}_{2}$. Molecular emission was collected as integrated over the whole plume that further reduced influence of any fractionation effects.

As it becomes clear from a calibration graph presented in Fig. 2, LAMIS can measure subnatural abundances of ${ }^{13} \mathrm{C}$ with sufficient precision. However, the decanoic acid powders used to prepare our reference samples consisted of microcrystal particles, and it was difficult to fully homogenize blended materials on a scale of the ablating laser spot size. Inaccuracies in preparing these reference mixtures increased the calibration errors. Better homogenized standards will improve precision and accuracy of LAMIS measurements. Previously, precision within 9\%o (interval $2 \sigma$ ) represented by relative standard deviation of $0.45 \%$ was achieved in LAMIS measurements of the boron isotopic ratio using a commercial sputtering target disk as a sample [33]. Precision can be further improved by accumulating a larger number of spectra with their mathematical treatment and normalization.

To examine applicability of LAMIS for geological analysis, we measured CN B ${ }^{2} \Sigma^{+} \rightarrow \mathrm{X}^{2} \Sigma^{+}$ $(\Delta v=+1)$ emission bands from several samples including coal, graphite and diamond. The results are shown in Fig. 3 in the same spectral interval and resolution as the data in Fig. 1 to facilitate their comparison. Spectra of the coal and graphite samples included atomic lines of metals, particularly $\mathrm{Fe}$ and $\mathrm{Cr}$. Measurements were performed using acquisition delay of $10 \mu$ s and gate 
width $50 \mu$ s, which was a longer delay than that used for measuring the spectra shown in Fig. 1. A long delay was effective in reducing intensity of atomic emission relative to intensity of the $\mathrm{CN}$ bands. However, molecular emission also decreased at delays longer than $10 \mu \mathrm{s}$. Coal from China exhibited relatively larger content of $\mathrm{Fe}$, while coal from Pittsburgh had relatively more Cr. South African coal standard SARM-18 contained relatively lower amount of both Fe and Cr.

These atomic lines of $\mathrm{Fe}$ and $\mathrm{Cr}$ shown in Fig. 3 did not interfere with the $\mathrm{B} \rightarrow \mathrm{X}(1-0)$ band head of minor isotopologue ${ }^{13} \mathrm{C}^{14} \mathrm{~N}$. To visualize the spectral position of the latter isotopic band we prepared a pressed pellet of $5 \%{ }^{13} \mathrm{C}$ amorphous carbon mixed with natural graphite powder. The spectrum of this carbon pellet is included in Fig. 3 (red trace) showing the isotopic band $\mathrm{B} \rightarrow \mathrm{X}$ (1-0) along with the same non-interfering contamination by elements $\mathrm{Fe}$ and $\mathrm{Cr}$. Spectrum of diamond was free from metal lines and exhibited pure $\mathrm{CN}$ emission bands. The results presented in Fig. 3 confirmed that consistent $\mathrm{CN}$ emission spectra can be obtained from different samples. The pelletized standard SARM-18 was properly homogeneous, while chunks of coal from Pittsburgh were not. Nevertheless, all tested samples yielded useful for LAMIS spectra.

It is technically possible to build a calibrated PLSR model for isotopic analysis of coal similar to that shown in Fig. 2 for decanoic acid. However, proper preparation of reference samples will be challenging. Occurrence of the atomic lines of metals within the spectral region of $\mathrm{CN}$ bands will further hamper the quality of PLSR procedure. An algorithm that automatically fits a Lorentzian profile to every foreign spectral line and digitally subtracts them from the spectrum can be applied as a remedy. Such a procedure can work relatively well if intensity of 
the atomic lines is no more than three times higher than the main spectrum of interest. A different approach to quantification in LAMIS can also be applied by simulating molecular spectra. Spectra of both ${ }^{12} \mathrm{C}^{14} \mathrm{~N}$ and ${ }^{13} \mathrm{C}^{14} \mathrm{~N}$ isotopologues can be synthesized numerically and then fitted to the experimental spectrum. A similar approach was used for studying kinetics of $\mathrm{C}_{2}$ isotopologues in laser ablation plasma from coal and solid organic samples $[9,10,14]$.

Emission spectra of diatomic carbon molecules $\mathrm{C}_{2}$ and their isotopologues were thoroughly studied by a large number of researchers. Several advanced spectrum simulation algorithms were recently developed and the results were published [34-36]. Utilization of $\mathrm{C}_{2}$ emission for LAMIS measurements was previously described $[7-10,14]$. One of the important parts of the $\mathrm{C}_{2}$ spectrum in LAMIS is where the band head of ${ }^{13} \mathrm{C}^{12} \mathrm{C} \mathrm{d} \mathrm{d}^{3} \Pi_{\mathrm{g}} \rightarrow \mathrm{a}^{3} \Pi_{\mathrm{u}}(1-0)$ at $474.45 \mathrm{~nm}$ appears among other lines of the Swan system of the most abundant isotopologue ${ }^{12} \mathrm{C}_{2}$. However, this part of ${ }^{12} \mathrm{C}_{2}$ spectrum between $473.8-476.5 \mathrm{~nm}$ was not synthesized or measured in previous studies with accuracy needed for LAMIS. We used our experimental spectra of $\mathrm{C}_{2}$ and previously calculated results [34-36] as a guidance to improve simulation of the $C_{2}$ spectrum, particularly around $474.45 \mathrm{~nm}$.

Synthetic spectrum of $\mathrm{C}_{2} \quad \mathrm{~d}^{3} \Pi_{\mathrm{g}} \rightarrow \mathrm{a}^{3} \Pi_{\mathrm{u}}$ transitions (Swan system) was computed within Herzberg's framework of diatomic molecules, similar to our earlier studies $[7,9,10]$. Spectral positions of the rotational lines, their energy levels, statistical weights and transition probabilities for triplet branches $\mathrm{P}, \mathrm{Q}$, and $\mathrm{R}$ of the ${ }^{12} \mathrm{C}_{2}(1-0)$ and ${ }^{13} \mathrm{C}^{12} \mathrm{C}(1-0)$ bands were directly taken from Brooke et al [34] and Ram et al [35], respectively. Rotational energy levels and transition probabilities for the rotational lines of the tails of triplet ${ }^{12} C_{2}$ branches $R(0-0)$ and $R(1-1)$ with 
high rotational quantum numbers up to $\mathrm{J}^{\prime}=110$ of the upper level and $\mathrm{J}^{\prime \prime}=109$ of the lower level were extrapolated from the data of Brooke et al [34]. However, spectral positions of the rotational lines in these tails were measured in our experiments and then included into our spectrum simulation.

The 3 members of triplet branch lines $\mathrm{R}_{1,2,3}$ in a tail of ${ }^{12} \mathrm{C}_{2}(0-0)$ coincided with each other at our resolution $(10 \mathrm{pm})$. Each triplet was considered as a single line. For example, the last measured line of branch $R_{1,2,3}(0-0)$ consisted of 3 coincident members: $R_{1}\left(J^{\prime \prime}=109\right), R_{2}\left(J^{\prime \prime}=108\right)$, and $R_{3}\left(J^{\prime \prime}=107\right)$ situated at $474.02 \mathrm{~nm}$ in air. The members of triplet lines $R_{1,2,3}(1-1)$ in the tail overlapped but not exactly coincided. Our experimental spectral resolution was insufficient to determine precise positions of individual members of the triplets but we measured the center wavelengths. The last measured line of branch $R_{1,2,3}(1-1)$ consisted of 3 members: $R_{1}(J "=106)$, $R_{2}(J "=105)$, and $R_{3}(J "=104)$ situated close to each other with the center at $474.30 \mathrm{~nm}$. However, the previous triplet with overlapping members $R_{1}\left(J^{\prime \prime}=105\right), R_{2}\left(J^{\prime \prime}=104\right)$, and $R_{3}(J "=103)$ centered at $474.80 \mathrm{~nm}$ was noticeably wide, hence we artificially split the 3 members by $\pm 7 \mathrm{pm}$ from the center to fit the experimental bandwidth of this convoluted triplet.

Spectral positions of the rotational lines in the Swan band (9-8) of ${ }^{12} \mathrm{C}_{2}$ were measured by Tanabashi et al [37] and earlier by Phillips and Davis [38]. An agreement between these two papers in line positions was satisfactory for our resolution. However, some of the lines were assigned differently and some lines were missing (never measured), particularly due to strong perturbations in the triplet branch $\mathrm{P}_{1,2,3}(9-8)$. We plotted the difference between observed (Tanabashi et al [37]) and calculated (Brooke et al [34]) line positions versus rotational quantum 
number $\mathrm{J}^{\prime \prime}$ in the three sub-branches $\mathrm{P}_{1,2,3}(9-8)$ of this band. Such plots allowed us to visualize perturbations that were not included in calculations of Brooke et al [34], and then to interpolate the probable positions of the missing lines. Locations of 7 interpolated lines of branches $\mathrm{P}_{1,2,3}(9$ 8 ) in the immediate vicinity of band head ${ }^{13} \mathrm{C}^{12} \mathrm{C}(1-0)$ were then manually adjusted to fit our experimental spectrum.

In order to remove overlapping and interfering lines of band ${ }^{13} \mathrm{C}^{12} \mathrm{C}(1-0)$ from the spectrum of band ${ }^{12} \mathrm{C}_{2}(9-8)$, we recorded this spectral interval in emission produced by ablating a glucose sample that was depleted in isotope ${ }^{13} \mathrm{C}$. The latter spectrum of ${ }^{12} \mathrm{C}_{2}$ is presented in Fig. 4 along with the spectrum from graphite of natural ${ }^{13} \mathrm{C} /{ }^{12} \mathrm{C}$ abundances, and our final simulation results. Graphite was ablated in a helium flow to eliminate any possibility of interference from $\mathrm{CN}$ molecule that cannot form in absence of nitrogen. Additionally, Doppler and collisional broadening of spectral lines is lower in helium relative to ablation in air or argon. These experiments were performed using Horiba JY-1250M spectrograph. Glucose spectrum was of relatively lower quality due to micro-inhomogeneity of the pellet but it clearly lacked ${ }^{13} \mathrm{C}^{12} \mathrm{C}$ and showed that no unknown lines of ${ }^{12} \mathrm{C}_{2}$ were directly under the band head of ${ }^{13} \mathrm{C}^{12} \mathrm{C}$. The energy levels, statistical weights and transition probabilities for the triplet branch $\mathrm{P}_{1,2,3}(9-8)$ used in our simulation were taken from Brooke et al [34].

In simulation of this spectrum, population of the rotational energy levels was assumed to obey Boltzmann distribution with rotational temperature as a fitting parameter common for all energy levels, branches, bands and isotopologues. However, intensity of each band and branch was assigned an individual fitting coefficient in order to account for the combined effect of 
different concentrations of the two isotopologues, possible differences in vibrational temperature and possible deviations from equilibrium in the ablation plasma. The best fit to an experimental spectrum was achieved with rotational temperature of $5155 \mathrm{~K}$. The final simulated sum of the five branches and their separate contributions to the spectrum are presented in Fig. 5. The intensity scale in this figure is normalized on the maximum of the band head of ${ }^{12} \mathrm{C}_{2} \mathrm{~d}^{3} \Pi_{\mathrm{g}} \rightarrow \mathrm{a}^{3} \Pi_{\mathrm{u}}$ $(1-0)$.

The results displayed in Fig. 4 and Fig. 5 demonstrated that carbon isotope ratio ${ }^{13} \mathrm{C} /{ }^{12} \mathrm{C}$ can be precisely determined through fitting a simulated spectrum to the experimental one in LAMIS. In the future, branch R of band ${ }^{12} \mathrm{C}_{2}(2-2)$ and possibly branch $\mathrm{P}$ of band ${ }^{12} \mathrm{C}_{2}(0-0)$ can also be included into a synthetic spectrum of the Swan system. This will improve the quality of both simulation and fitting. However, even now using the presented simulation and fitting procedure, we obtained a ${ }^{13} \mathrm{C} /{ }^{12} \mathrm{C}$ isotopic ratio of $1.08 \%$ for the graphite sample that we used. This ratio agrees very well with the value of $1.0804 \%$ measured from this sample by isotope ratio mass spectrometry (IRMS).

\section{CONCLUSION}

Physical possibilities were illustrated for measuring carbon isotopes in coal and other natural samples utilizing $\mathrm{CN}$ or $\mathrm{C}_{2}$ spectra with the LAMIS technique. During laser ablation of carboncontaining samples, both $\mathrm{CN}$ and $\mathrm{C}_{2}$ radicals are often generated in comparable quantities. Accordingly, both these species were used for measuring carbon isotopic ratio. Two approaches 
to quantification in LAMIS were established: (1) empirical calibration of spectra using a set of reference standards and (2) numerical fitting of a simulated spectrum to the experimental one.

Rapid spatial and temporal evolution of the plasma plumes during laser ablation may cause kinetic fractionation effects altering stoichiometric and isotopic equilibrium between the plasma phase and the original solid sample. For the purpose of LAMIS, it is important to choose experimental conditions at which the ablated material becomes completely atomized, then equilibrates and associates into excited diatomic molecules. Plausibly, the use of the forthharmonic laser ablation at $266 \mathrm{~nm}$ will be preferable over the presently used ablation at $1064 \mathrm{~nm}$. Operating at longer delays facilitates equilibration within the plume, so that the measured isotopic ratio approaches the real ratio in the sample roughly at the same time when molecular emission is also reaching its maximum.

Formation of $\mathrm{CN}$ in our experiments was less prone to isotope fractionation compared to $\mathrm{C}_{2}$. Emission was collected from the whole plume to reduce fractionation effects. Empirical calibration based on reference standards minimized any possible errors attributed to remaining latent fractionation in the ablation plumes. In the future, $\mathrm{CN}$ emission can be utilized for simultaneous determination of ${ }^{13} \mathrm{C}$ and ${ }^{15} \mathrm{~N}$ isotopes. Therefore, LAMIS promises to be a useful technique in coal, oil and shale exploration, carbon sequestration monitoring, agronomy studies and many other fields. The main advantages include rapid analysis at atmospheric pressure, without any preparation of original unaltered samples. LAMIS instrumentation is transportable, and consequently field measurements are possible. 
Recent introduction of the femtosecond LAMIS $[39,40]$ indicated further prospects for improving accuracy and sensitivity in this technique. Femtosecond ablation atomizes materials better than nanosecond pulses do, thus reducing chances for unwanted fractionation effects. Femtosecond laser plasma is cooler than that in nanosecond ablation, which results in both lower continuum background and enhanced rates for molecular association (after complete atomization of the ablated material) versus corresponding rates in nanosecond laser plasma. At the same laser power delivered to the sample, LAMIS signal from femtosecond ablation was 6fold stronger than that from nanosecond ablation. Femtosecond ablation does not significantly heat up the sample, and therefore the probability of vaporizing specific native molecular fragments directly from the sample surface is greatly diminished. The latter increases the degree of isotopic exchange and equilibration in the plasma plume, and thus accuracy of LAMIS measurements.

\section{ACKNOWLEDGMENTS}

This research was funded through SBIR program by the U.S. Department of Energy with the Award No. DE-SC0007546 granted to Applied Spectra, Inc. The work at the Lawrence Berkeley National Laboratory was supported by the Defense Nuclear Nonproliferation Research and Development Office and the Office of Basic Energy Sciences of the U.S. Department of Energy under contract number DE-AC02-05CH11231.

\section{REFERENCES}


[1]. R. Bibby, S. Widdicombe, H. Parry, J. Spicer, R. Pipe, Effects of ocean acidification on the immune response of the blue mussel Mytilus edulis, Aquat. Biol., 2 (2008) 67-74.

[2]. S. Solomon, D. Qin, M. Manning, Z. Chen, M. Marquis, K.B. Averyt, M. Tignor, H.L. Miller, "IPCC, Climate Change 2007: The Physical Science Basis. Contribution of Working Group I to the Fourth Assessment Report of the Intergovernmental Panel on Climate Change," Cambridge University Press, Cambridge, (2007) 98-101.

[3]. "2010 carbon sequestration Atlas of the United States and Canada," 3rd Edition (Atlas III), http://www.netl.doe.gov/KMD/CDs/atlasIII/2010atlasIII.pdf

[4]. J. Jain, D. McIntyre, K. Ayyalasomayajula, V. Dikshit, C. Goueguel, F. Yu-Yueh, J. Singh, Application of laser-induced breakdown spectroscopy in carbon sequestration research and development, Pramana-J. Phys., 83 (2014) 179-188.

[5]. G. Johnson, M. Raistrick, B. Mayer, M. Shevalier, S. Taylor, M. Nightingale, I. Hutcheon, The use of stable isotope measurements for monitoring and verification of $\mathrm{CO}_{2}$ storage, Energy Procedia, 1 (2009) 2315-2322.

[6]. A. Bechtel, R. Gratzer, R. F. Sachsenhofer, J. Gusterhuber, A. Lücke, W. Püttmann, Biomarker and carbon isotope variation in coal and fossil wood of Central Europe through the Cenozoic, Palaeogeography, palaeoclimatology, palaeoecology 262 (2008) 166-175.

[7]. R.E. Russo, A.A. Bol'shakov, X. Mao, C.P. McKay, D.L. Perry, O. Sorkhabi, Laser ablation molecular isotopic spectrometry, Spectrochim. Acta Part B, 66 (2011) 99-104.

[8]. A.A. Bol'shakov, X. Mao, D.L. Perry, R.E. Russo, Laser ablation molecular isotopic spectrometry for rare isotopes of the light elements, Spectrosc., 29, no.6, (2014) 30-39. 
[9]. M. Dong, G.C.-Y. Chan, X. Mao, J.J. Gonzalez, J. Lu, R.E. Russo, Elucidation of $\mathrm{C}_{2}$ and CN formation mechanisms in laser-induced plasmas through correlation analysis of carbon isotopic ratio, Spectrochim. Acta Part B, 100, (2014) 62-69.

[10]. M. Dong, X. Mao, J.J. Gonzalez, J. Lu, R.E. Russo, Carbon isotope separation and molecular formation in laser-induced plasmas by Laser Ablation Molecular Isotopic Spectrometry, Anal. Chem., 85 (2013) 2899-2906.

[11]. A.A. Bol'shakov, J.H. Yoo, C. Liu, J. R. Plumer, R.E. Russo, Laser-induced breakdown spectroscopy in industrial and security applications, Appl. Opt., 49 (2010) C132-C142.

[12]. V.M. Nemets, I.A. Rodyushkin, A.A. Solov'ev, V.N. Funtov, Isotopic carbon analysis using the $\mathrm{C}_{2}$ molecule spectrum, J. Appl. Spectrosc., 52 (1990) 461-465.

[13]. G.S. Lazeyeva, V.M. Nemetz, A.A. Petrov, Spectral-isotopic method of determination of gas- forming elements in organic and inorganic substances, Spectrochim. Acta Part B, 36 (1981) $1233-1242$.

[14]. M. Dong, X. Mao, J.J. Gonzalez, J. Lu, R.E. Russo, Time-resolved LIBS of atomic and molecular carbon from coal in air, argon and helium, J. Anal. At. Spectrom., 27 (2012) 2066-2075.

[15]. X. Li, Z. Wang, Y. Fu, Z. Li, J. Liu, W. Ni, Application of a spectrum standardization method for carbon analysis in coal using laser-induced breakdown spectroscopy (LIBS), Appl. Spectrosc., 68 (2014) 955-962

[16]. L. St-Onge, R. Sing, S. Béchard, M. Sabsabi, Carbon emissions following $1.064 \mu \mathrm{m}$ laser ablation of graphite and organic samples in ambient air. Appl. Phys. A, 69, Suppl. 1 (1999) S913-S916. 
[17]. M. Baudelet, M. Boueri, J. Yu, S.S. Mao, V. Piscitelli, X. Mao, R.E. Russo, Time-resolved ultraviolet laser-induced breakdown spectroscopy for organic material analysis, Spectrochim. Acta Part B, 62 (2007) 1329-1334.

[18]. J. Serrano, J. Moros, J.J. Laserna, Sensing signatures mediated by chemical structure of molecular solids in laser-induced plasmas, Anal. Chem., 87 (2015) 2794-2801.

[19]. W.Q. Lei, Q.L. Ma, V. Motto-Ros, X.S. Bai, L.J. Zheng, H.P. Zeng, J. Yu, Effect of ablation photon energy on the distribution of molecular species in laser-induced plasma from polymer in air, Spectrochim. Acta Part B, 73 (2012) 7-12.

[20]. A.V. Gusarov, V.I. Titov, W. Scharff, Gas-kinetic simulation of carbon vapour molecular composition at nanosecond laser ablation of graphite in vacuum, J. Phys. D, 38 (2005) 28812889.

[21]. Y. Iida, E.S. Yeung, Optical monitoring of laser-induced plasma derived from graphite and characterization of the deposited carbon film, Appl. Spectrosc., 48 (1994) 945-950.

[22]. V.S. Burakov, A.F. Bokhonov, M.I. Nedel'ko, N.A. Savastenko, N.V. Tarasenko, Dynamics of the emission of light by $\mathrm{C}_{2}$ and $\mathrm{C}_{3}$ molecules in a laser plasma produced by twopulse irradiation of the target, J. Appl. Spectrosc., 69 (2002) 907-912.

[23]. S.-B. Wen, X. Mao, R. Greif, R.E. Russo, Expansion of the laser ablation vapor plume into a background gas. I Analysis, J. Appl. Phys., 101 (2007) 023114, 1-13.

[24]. V.A. Trofimov, I.A. Shirokov, Computer simulation of expansion of a carbon laser plasma after ablation in nitrogen atmosphere, Tech. Phys., 54 (2009) 974-980.

[25]. S.V. Shabanov, I.B. Gornushkin, Two-dimensional axisymmetric models of laser induced plasmas relevant to laser induced breakdown spectroscopy, Spectrochim. Acta Part B, 100 (2014) 147-172. 
[26]. G.M. Fuge, M.N.R. Ashfold, S.J. Henley, Studies of the plume emission during the femtosecond and nanosecond ablation of graphite in nitrogen, J. Appl. Phys, 99 (2006) 014309, $1-12$.

[27]. S.M. Park, K.H. Lee, Angular distributions of $\mathrm{C}_{2}$ and $\mathrm{CN}$ formed by laser ablation of graphite in a nitrogen atmosphere, Appl. Surf. Sci., 178 (2001) 37-43.

[28]. K.F. Al-Shboul, S.S. Harilal, A. Hassanein, Gas dynamic effects on formation of carbon dimers in laser-produced plasmas, Appl. Phys. Lett., 99 (2011) 131506, 1-3.

[29]. Q. Ma, P.J. Dagdigian, Kinetic model of atomic and molecular emissions in laser-induced breakdown spectroscopy of organic compounds, Anal. Bioanal. Chem. 400 (2011) 31933205 .

[30]. A.A. Bol'shakov, X. Mao, C.P. McKay, R.E. Russo, Laser ablation - optical cavity isotopic spectrometer for Mars rovers, Proc. SPIE, 8385 (2012) 83850C, 1-7; DOI:10.1117/12.919905 [31] C. Sneden, S. Lucatello, R.S. Ram, J.S.A. Brooke, P. Bernath, Line lists for the $\mathrm{A}^{2} \Pi-\mathrm{X}^{2} \Sigma^{+}$ (red) and $\mathrm{B}^{2} \Sigma^{+}-\mathrm{X}^{2} \Sigma^{+}$(violet) systems of $\mathrm{CN},{ }^{13} \mathrm{C}^{14} \mathrm{~N}$, and ${ }^{12} \mathrm{C}^{15} \mathrm{~N}$, and application to astronomical spectra, Astrophys. J. Suppl. Ser., 214 (2014) paper 26, 1-10.

[32]. X. Mao, A.A. Bol'shakov, D.L. Perry, O. Sorkhabi, R.E. Russo, Laser Ablation Molecular Isotopic Spectrometry: Parameter influence on boron isotope measurements, Spectrochim. Acta Part B, 66 (2011) 604-609.

[33]. A. Sarkar, X. Mao, R.E. Russo, Advancing the analytical capabilities of laser ablation molecular isotopic spectrometry for boron isotopic analysis, Spectrochim. Acta Part B, 92 (2014) $42-50$. 
[34]. J.S.A. Brooke, P.F. Bernath, T.W. Schmidt, G.B. Bacskay, Line strengths and updated molecular constants for the $\mathrm{C}_{2}$ Swan system, J. Quant. Spectrosc. Radiat. Transf., 124 (2013) 1120.

[35]. R.S. Ram, J.S.A. Brooke, P.F. Bernath, C. Sneden, S. Lucatello, Improved line data for the Swan system ${ }^{12} \mathrm{C}^{13} \mathrm{C}$ isotopologue, Astrophys. J. Suppl. Ser., 211 (2014) paper 5, 1-7. [36]. C.G. Parigger, A.C. Woods, D.M. Surmick, G. Gautam, M.J. Witte, J.O. Hornkohl, Computation of diatomic molecular spectra for selected transitions of aluminum monoxide, cyanide, diatomic carbon, and titanium monoxide, Spectrochim. Acta Part B, 107, (2015) 132138.

[37]. A. Tanabashi, T. Hirao, T. Amano. The Swan system of $\mathrm{C}_{2}$ : A global analysis of Fourier transform emission spectra, Astrophys. J. Suppl. Ser., 169 (2007) 472-484.

[38]. J.G. Phillips, S.P Davis, The Swan system of the $\mathrm{C}_{2}$ molecule. The spectrum of the $\mathrm{HgH}$ molecule, in "Berkeley Analyses of Molecular Spectra", Vol. 2, Univ. of California Press, Berkeley, 1968, 260 p.

[39]. B. Yee, K.C. Hartig, P. Ko, J. McNutt, I. Jovanovic, Measurement of boron isotopic ratio with non-gated molecular spectroscopy of femtosecond laser-produced plasma, Spectrochim. Acta Part B, 79-80, (2013) 72-76.

[40]. H. Hou, G.C.-Y. Chan, X. Mao, V. Zorba, R. Zheng, R.E. Russo, Femtosecond laser ablation molecular isotopic spectrometry for zirconium isotope analysis, Anal. Chem., 87 (2015) $4788-4796$.

\section{FIGURE CAPTIONS}


Fig. 1. $\mathrm{CN}$ vibrational bands $(\mathrm{B} \rightarrow \mathrm{X} ; \Delta v=1)$ formed during ablation of natural graphite and enriched benzamide pellets. Time gate width was $40 \mu$ s with a delay $2 \mu$ s. Spectra averaged over 100 laser pulses.

Fig. 2. Calibration plot with a linear fit and $95 \%$ confidence interval for the ${ }^{13} \mathrm{C}$ isotopic content calculated using CN spectra in PLSR model.

Fig. 3. CN bands (same as in Fig.1) formed during ablation of three samples of coal, diamond and $5 \%{ }^{13} \mathrm{C}$-enriched graphite pellet. Time gate width was $50 \mu$ s with a delay $10 \mu \mathrm{s}$. Spectra averaged over 300 laser pulses.

Fig. 4. Part of the Swan system with band (1-0) of ${ }^{13} \mathrm{C}^{12} \mathrm{C}$ among rotational lines of ${ }^{12} \mathrm{C}_{2}$. Synthetic spectrum compared to spectra recorded during ablation of ${ }^{13} \mathrm{C}$-depleted glucose in argon and graphite of natural abundance in helium. Time gate width was $30 \mu$ s with a delay $2 \mu$ s. Spectra averaged over 1000 laser pulses.

Fig. 5. Components of the simulated part of the Swan system. Labels of the rotational lines of ${ }^{12} \mathrm{C}_{2}$ are simplified as following "R00-109" stands for 3 coincident lines: $\mathrm{R}_{1}(\mathrm{~J}=109)$, $\mathrm{R}_{2}(\mathrm{~J}=108)$, and $\mathrm{R}_{3}(\mathrm{~J}=107)$ of ${ }^{12} \mathrm{C}_{2}(0-0)$; "R11-106" stands for $\mathrm{R}_{1}(\mathrm{~J}=106), \mathrm{R}_{2}(\mathrm{~J}=105)$, and $\mathrm{R}_{3}\left(\mathrm{~J}^{\prime \prime}=104\right)$ of ${ }^{12} \mathrm{C}_{2}(1-1)$ 


\section{NOVELTY STATEMENT}

LAMIS provided the ability for determination of carbon isotopes at natural levels. Spectra of the

Swan system of ${ }^{12} \mathrm{C}_{2}$ and ${ }^{13} \mathrm{C}^{12} \mathrm{C}$ were synthesized around the band head of ${ }^{13} \mathrm{C}^{12} \mathrm{C}$ and used for quantification of isotopes. 


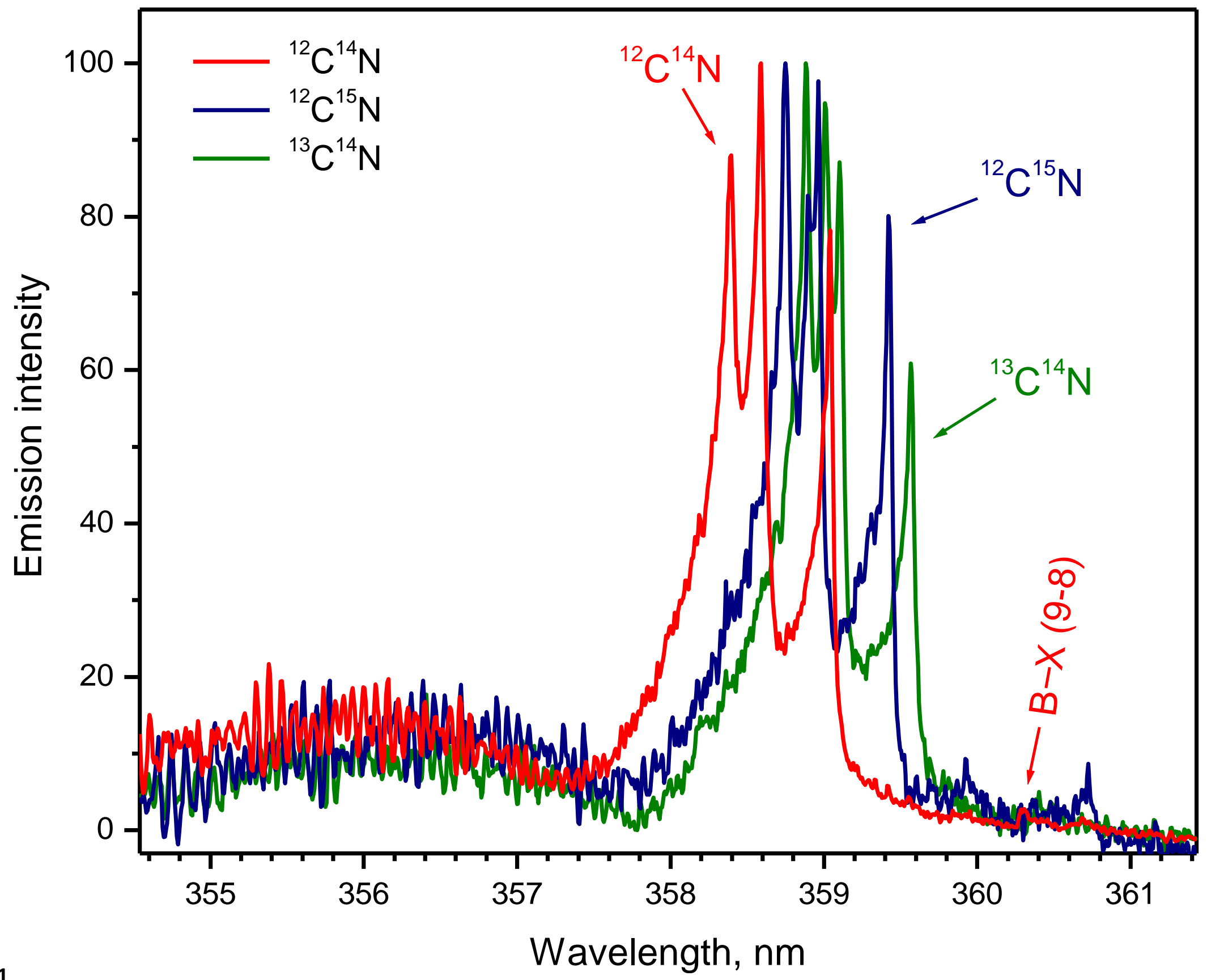

Fig. 1 


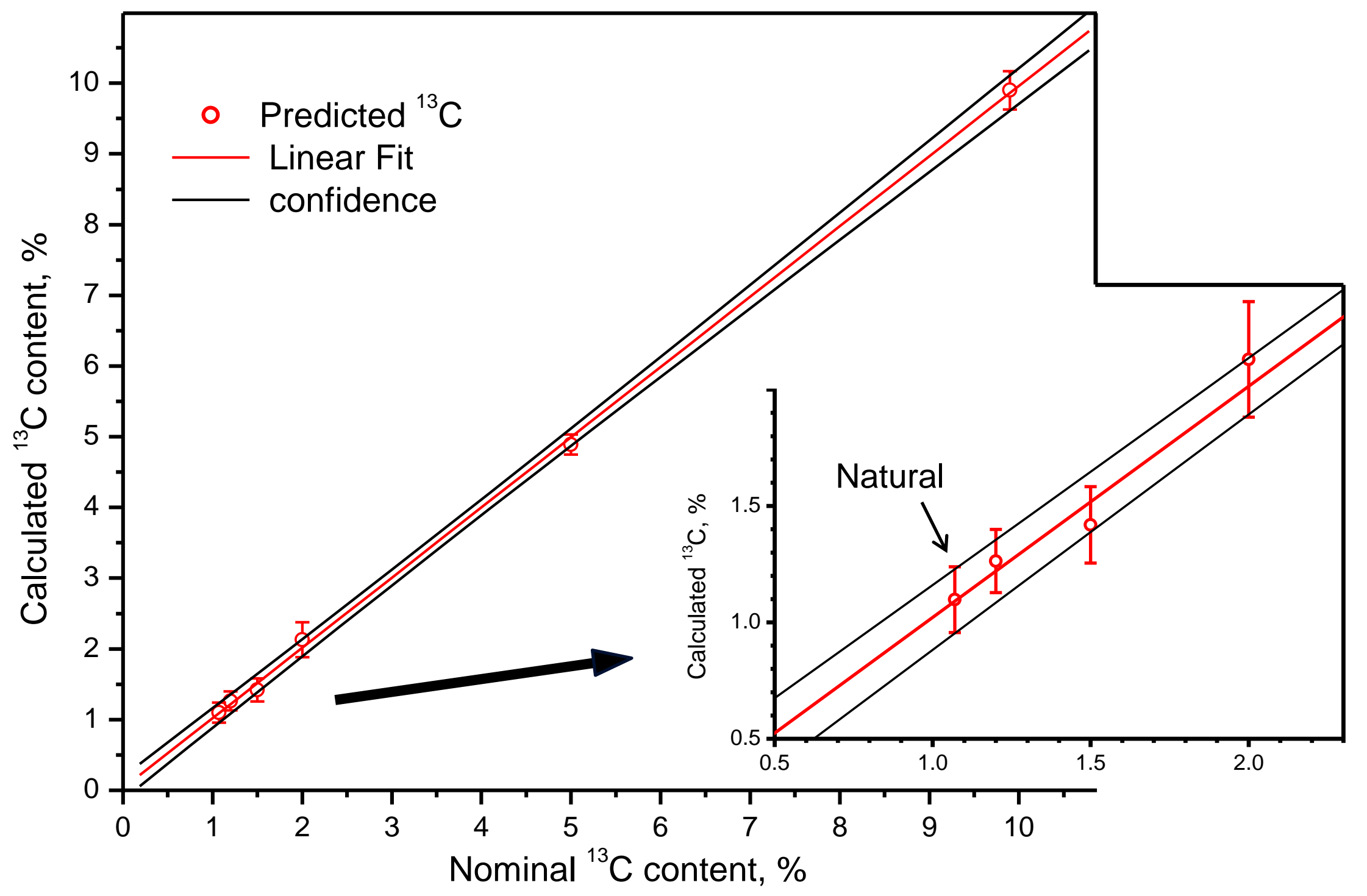

Fig. 2 


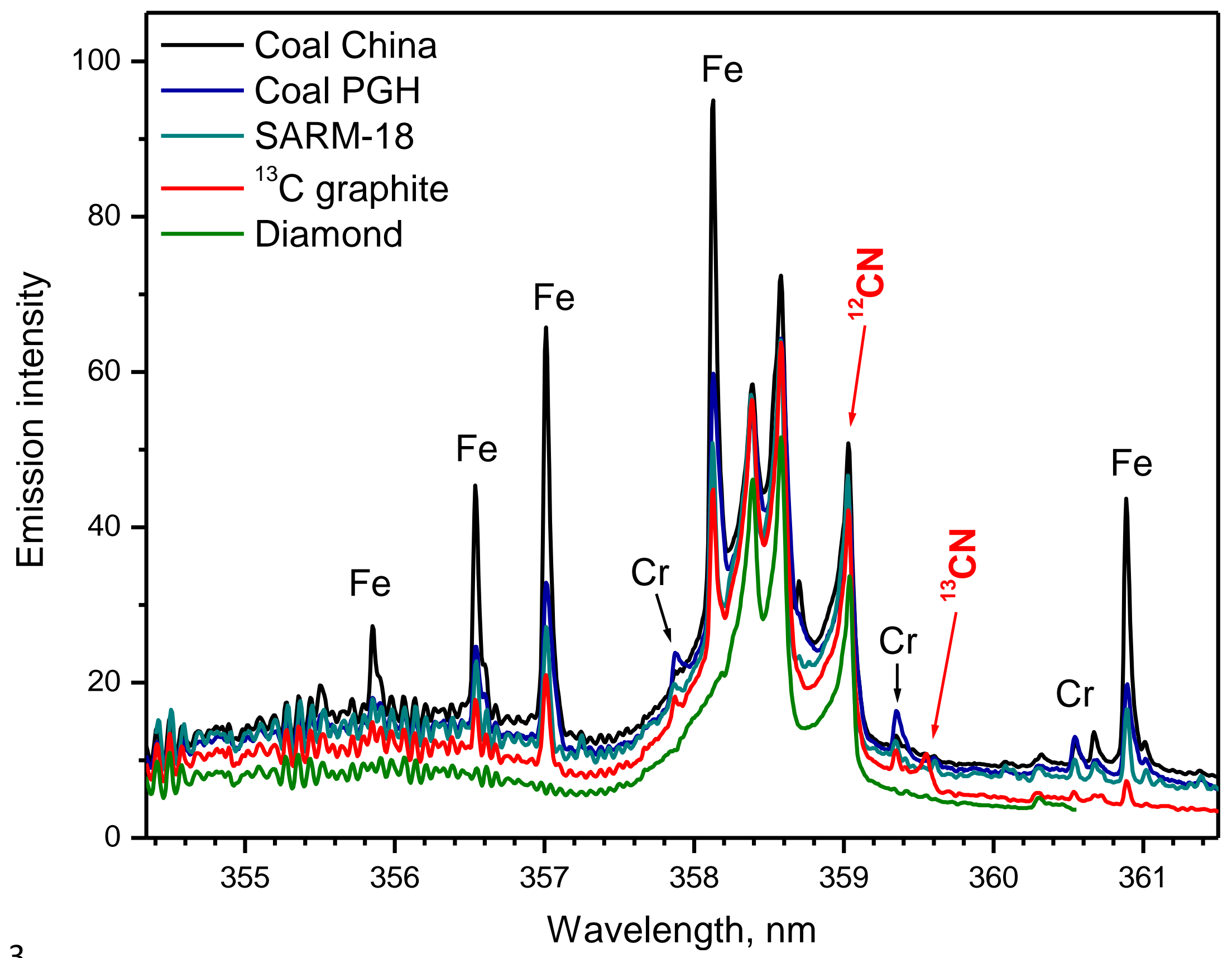

Fig. 3 


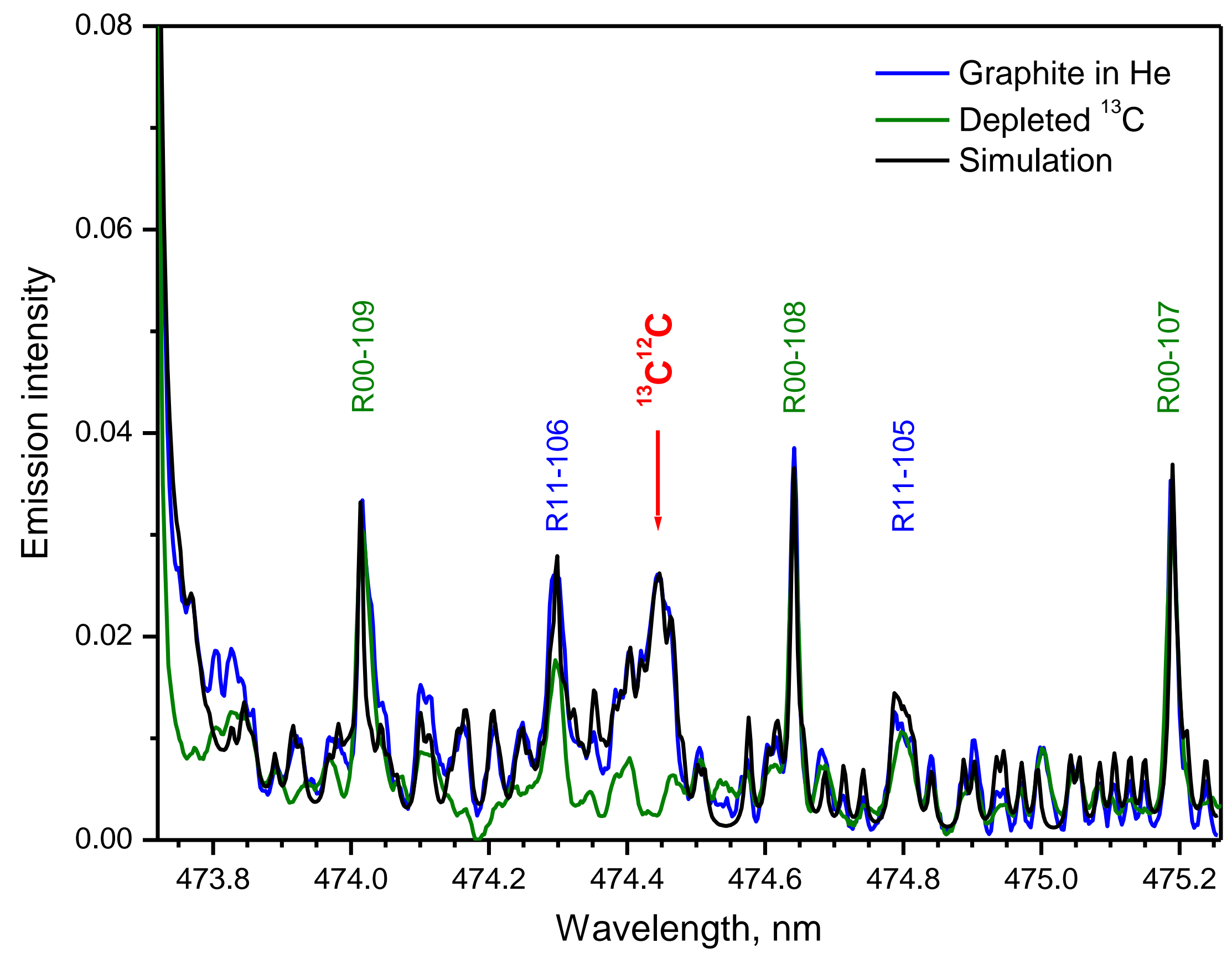

Fig. 4 


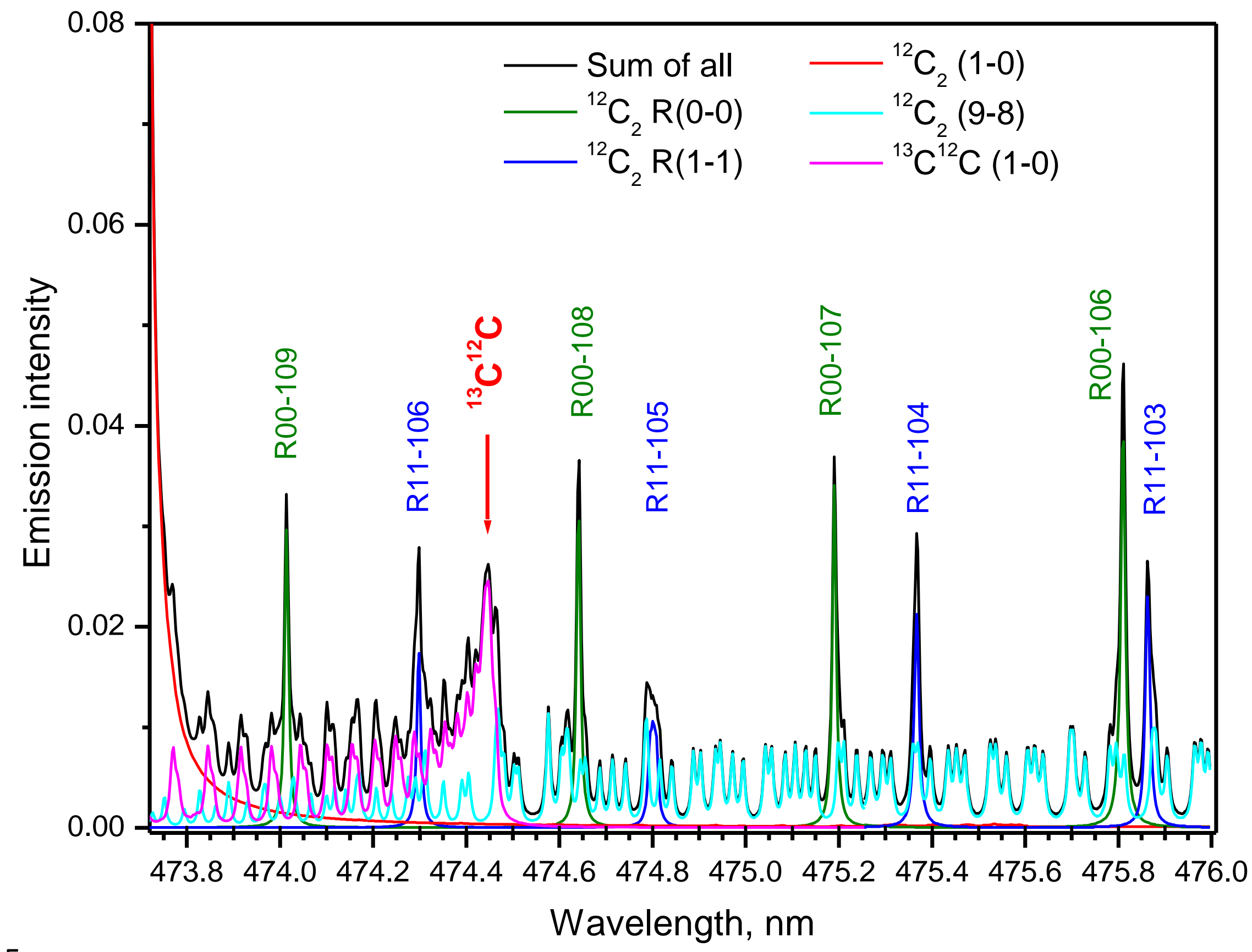

Fig. 5 